\title{
Extreme wave solutions: Parametric studies and wavelet analysis
}

\author{
Ayan Moitra ${ }^{\mathrm{a}}$, Christopher Chabalko $^{\mathrm{a}}$, Balakumar Balachandran $^{\mathrm{a}}$ \\ ${ }^{a}$ Department of Mechanical Engineering, University of Maryland, College Park, MD 20742-3035
}

\begin{abstract}
Wave fields for near homoclinic, single mode rogue-wave solutions of the periodic nonlinear Schrödinger equation are presented. Parameters of candidate solutions are estimated and refined through an eigenvalue solution procedure. An overview of the estimation and refining procedure used by the authors is provided. Solutions are scaled to facilitate experimental implementation. The continuous wavelet transform is used to carry out time-frequency analyses and the results obtained are demonstrative of the dispersion relation as well as the time varying side band energy transfer associated with the Benjamin-Feir instability. The analysis framework and approach used are validated with the Peregrine solution. Other extreme wave solutions are analyzed as well. The framework presented here could serve as a basis for experimental investigations into single mode rogue waves as well as other localizations in wave fields.
\end{abstract}

Keywords: nonlinear Schrödinger equation, rogue waves, graphics pocessing unit based computations, time-frequency analyses

\section{Introduction and background}

In the context of many systems, ranging from the ocean $[1,2]$ to optical fibers [3], extreme waves have been reported. These waves are characterized by extreme amplitudes, which can occur intermittently in time and space [4]. The Benjamin-Feir (modulational) instability [5] has been proposed as one of the mechanisms for rogue-wave formation [6]. Given that the nonlinear Schrödinger equation (NLSE) exhibits the Benjamin-Feir instability, the NLSE has been widely studied as a model for extreme wave behavior. Other models include the Dysthe, Kadomtsev Petviashvili (KP), and Korteweg de Vries (KdV) equations. While many solutions and solution families of the NLSE are known, experimental investigations have been limited. Several groups have carried out experiments, in which rogue waves have been provoked in wave tanks $[1],[2],[7]$. The typical focus of these experiments has been only on the rogue-wave amplitude. On the other hand, Onorato et al. [8],[9],[10] and Osborne [11] have performed laboratory experiments to investigate the presence of modulational instability and rogue wave modes in random wave spectra.

While infrequent, oceanic rogue waves are gaining attention due to their destructive nature. Lim- ited descriptive quantitative data has been recorded [12]. In this work, the authors present a method to determine the parameters of a family of single mode near homoclinic solutions to the NLSE. The first step in the procedure provides a map of eigenvalues which leads to potential solutions. This eigenvalue map may allow researchers to explore new solutions to the NLSE, including rogue-wave solutions. The solutions are derived from a pre-filtering (predictive) and eigenvalue solving (corrector) procedure for the NLSE, as detailed in a recent effort by the authors [13].

Here, for brevity, only a short review of some of the most significant results related to rogue-wave solutions to the NLSE is provided. Tracy [14] presented some of the first analytic solutions to the NLSE. Akhmediev and Korneev [15] determined a family of single parameter solutions. In studies of the nonlinear Schrödinger equation with periodic boundary conditions, homoclinic (or breather) solutions have been used to model rogue waves $[16,17]$. Islas and Schober [17] were the first to correlate the likelihood of rogue waves with the closeness to homoclinic solutions. Osborne [18] has contributed significant work on hyperfast modeling of rogue waves and presented an analysis of nonlinear Fourier modes of a wavefield, identifying rogue 
modes. The results have implications in other domains such as fiber optical cables as detailed in $[1,19]$. A comprehensive review of past contributions and the state of the art related to rogue waves can be found in several review papers (e.g., [6], [20], and $[21])$.

In the present work, the authors aim to advance the identification and analysis of rogue waves and soliton behavior of the NLSE. As detailed in a recent effort [13], the authors present a procedure, not available elsewhere in the literature, which allows for the identification of spectral parameters of single mode near homoclinic theta function based solutions to the NLSE. While this solution family is already known, examples of variations in the parameters governing the solutions are not readily available in the literature. Furthermore, connections between the features of the numerically generated eigenvalue space and the solutions appearing therein have been seldom highlighted. The eigenvalue map presented in this work provides a quantitatively accurate overview and context of the behavior of the solution space from which these solutions originate. The particular solutions presented here, and the insights provided by the mapping procedure, can substantially enhance the understanding and stimulate further investigations into NLSE solutions and associated rogue waves.

Finally, wavelet analyses of rogue-wave solutions is used to characterize evolution in the timefrequency domain. This analysis helps reveal the dispersion relation; that is, low frequency components are identified to travel faster than high frequency components of rogue waves. Furthermore, the time variation of the energy in the side bands associated with a Benjamin-Feir type instability is presented. This analysis is validated on the Peregrine solution and applied to other single mode rogue-wave solutions. It can be easily applied by other researchers to analyze simulated and experimental results. The Peregrine breather has been demonstrated experimentally in a water tank; however, this fact alone does not prove that hydrodynamic surface wave behavior is governed by the NLSE. In fact, the Peregrine solution is only one of many solutions to the NLSE and other wave equations. In order to more conclusively verify the governing model of surface waves, other NLSE solutions would need to be experimentally studied. The predictive results and analysis detailed in this effort can be used to facilitate such experimental efforts.

The remainder of this paper has been arranged as follows. An overview of the eigenvalue map is provided in the next section, along with illustrations of several features of the map. A solution similar to the Peregrine breather is demonstrated. Following this discussion, solutions quite different from the Peregrine breather are presented. In the next section, for a particular spectral parameter variation, the transition from a rogue wave to an exaggerated wave formation is shown. In the fifth section, solutions with physical scaling similar to water waves in published experimental investigations are presented. Finally, continuous wavelet analysis is applied to several solutions to reveal time-frequency behavior including the dispersion relation and the intermittent transfer of energy among the carrier frequency and side bands. Through the discussion presented in the different sections, the authors expect to demonstrate the utility of the solution generation procedure and analysis. Concluding remarks are collected and presented together at the end.

\section{Eigenvalue solution method}

\subsection{Candidate solutions}

A brief overview of the procedure to generate the map of candidate solutions to the NLSE is presented in this section. For further details and examples, the reader is referred to the group's previous effort [13]. The scaled NLSE, a dimensionless equation, takes the form

$$
i u_{T}-u_{X X}+2 \sigma|u|^{2} u=0
$$

where $u(X, T)$ is the complex wave envelope field with periodic boundary condition $u(X, T)=u(X+$ $L, T)$ for $0 \leq X \leq L, T$ is time, $X$ is the spatial variable, $i=\sqrt{-1}$, and the subscripts indicate the associated partial derivatives. The focusing case requires $\sigma=-1$ while $\sigma=1$ yields the defocusing case. Space periodic spectral solutions to the NLSE can be described as

$$
u(X, T)=A \frac{\Theta\left(X, T \mid \tau, \delta^{-}\right)}{\Theta\left(X, T \mid \tau, \delta^{+}\right)} e^{2 i A^{2} T} .
$$

where $\Theta\left(X, T \mid \tau, \delta^{ \pm}\right)$is a Riemann theta function [14], [22], and [23]. A single unstable mode $(N=2)$ is considered by expressing $\Theta\left(X, T \mid \tau, \delta^{ \pm}\right)$as a twodimensional theta function defined as 


$$
\begin{aligned}
& \Theta\left(X, T \mid \tau, \delta^{ \pm}\right)=\sum_{m_{1}=-\infty}^{\infty} \sum_{m_{2}=-\infty}^{\infty} \exp i\left[\sum_{n=1}^{2} m_{n} K_{n} X+\right. \\
& \left.\sum_{n=1}^{2} m_{n} \Omega_{n} T+\sum_{n=1}^{2} m_{n} \delta_{n}^{ \pm}+\sum_{j=1}^{2} \sum_{k=1}^{2} m_{j} m_{k} \tau_{j k}\right]
\end{aligned}
$$

The parameters governing the theta function $\left(K_{n}, \Omega_{n}\right.$, and $\left.\delta^{ \pm}\right)$are defined in terms of $A, \lambda_{R}$, $\lambda_{I}, \epsilon_{0}$, and $\theta$, which are referred to as spectral parameters of the near homoclinic solution. Following the notation used in the literature [11], the spectral parameters are defined as

$$
\begin{gathered}
\epsilon_{1}=\epsilon_{0} e^{i \theta}, \epsilon_{2}=\epsilon_{1}^{*}, \sigma_{1}=1, \sigma_{2}=-1 \\
\lambda_{1}=\lambda_{R}+i \lambda_{I}, \quad \lambda_{2}=\lambda_{1}^{*} \\
K_{n}=-2 \sqrt{A^{2}+\lambda_{n}^{2}}, \quad \Omega_{n}=2 \lambda_{n} K_{n} \\
\delta_{n}^{ \pm}=\pi+i \ln \left(\lambda_{n} \mp \frac{1}{2} K_{n}\right)+i \ln \left(\sigma_{n} \lambda_{n}-(-1)^{n} \frac{1}{2} K_{n}\right) \\
\tau_{11}=\frac{1}{2}+\frac{i}{\pi} \ln \left(\frac{K_{1}^{2}}{\epsilon_{1}}\right), \quad \tau_{22}=\frac{1}{2}+\frac{i}{\pi} \ln \left(\frac{K_{2}^{2}}{\epsilon_{2}}\right) \\
\tau_{12}=\tau_{21}=\frac{i}{2 \pi} \ln \left(\frac{1+\lambda_{1} \lambda_{2}+\frac{1}{4} K_{1} K_{2}}{1+\lambda_{1} \lambda_{2}-\frac{1}{4} K_{1} K_{2}}\right)
\end{gathered}
$$

Initially, the solution space is defined with $A=1$, $\theta=0$, and $\epsilon_{0} \leq 0.05$. These parameters are refined in a subsequent step leaving $\lambda_{R}$ and $\lambda_{I}$ as the only free parameters governing the initial function selection. Potential successful parameter combinations are determined by evaluating the periodicity of $u(X, 0)$ over an interval $n L$ (where $n=1,2,3$ ) for a given $\left(\lambda_{R}, \lambda_{I}\right)$. The periodicity of all solutions is tested over the entire two-dimensional grid of $\left(\lambda_{R}, \lambda_{I}\right)$ values. The periodicity associated with each combination of $\left(\lambda_{R}, \lambda_{I}\right)$ is estimated independently and thus carried out in parallel via a graphics processing unit implementation, as detailed in the authors' recent work [13]. The resulting map of $\left(\lambda_{R}, \lambda_{I}\right)$ pairs which form periodic $u(X, T)$ functions with $L=12$ is shown in Figure 1. Periodic

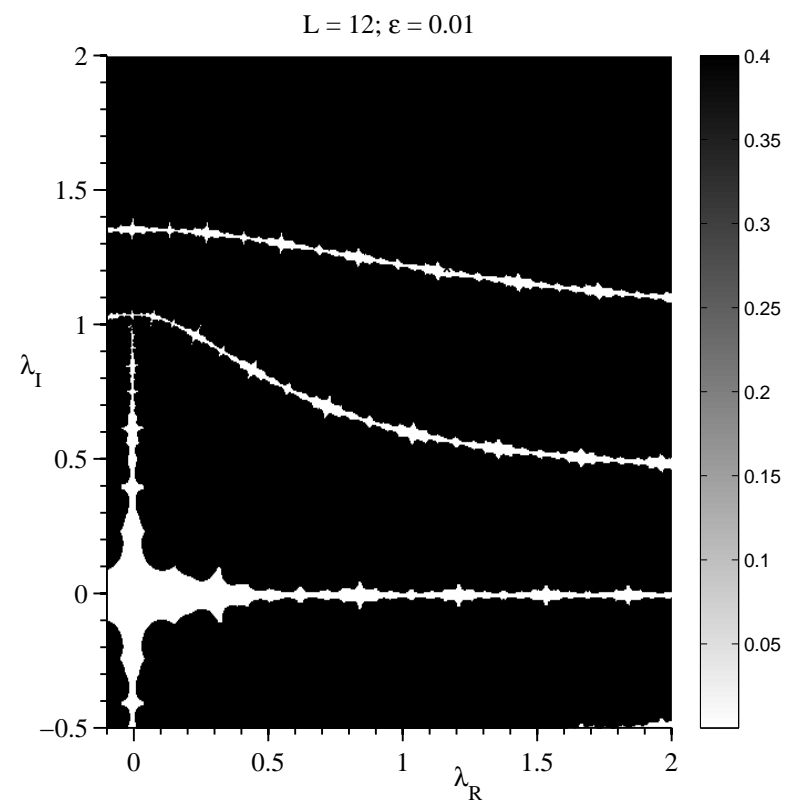

Figure 1: Map of parameters governing periodic Riemann theta functions as defined in Eq. (2). Light colored locations indicate periodic functions over the interval $L$.

functions, and hence parameter values of potential solutions, are displayed in white on the candidate map. The dark regions indicate $\left(\lambda_{R}, \lambda_{I}\right)$ pairs which do not result in periodic functions, and hence are not guaranteed to be solutions. If one eliminates these regions and focuses on the light regions, one is guaranteed to find solutions to the NLSE, enables more efficient parameter space exploration. Map variations with respect to $L$ is described in a later section.

\subsection{Eigenvalue solution}

Given a particular choice of $\left(\lambda_{R}, \lambda_{I}\right)$ from the map described above, the spectral parameters of a near homoclinic solution can be determined by solving the following spectral eigenvalue problem:

$$
\Psi_{x}=Q(\lambda) \Psi, \quad Q=\left(\begin{array}{cc}
-i \lambda & u \\
\sigma u^{*} & i \lambda
\end{array}\right)
$$

The eigenvalue solution procedure closely follows prior work $[11,13]$. The eigenvalue problem of Eq. (9) is recast as a Floquet problem and a constant potential is used to determine the spectral eigenfunction over a given interval. The monodromy 
matrix is given by [24]

$$
T\left(x_{0}, \lambda\right)=\prod_{j=M}^{0} U\left(u_{j}, \lambda\right)
$$

Finally, the main spectrum eigenvalues, $\lambda_{k}$ for $k=1,2, \ldots, 2 N$, are determined from the trace of the monodromy matrix.

The parameters $\epsilon$ and $\theta$ are determined from the pair of eigenvalues, which are close to the single complex eigenvalue used to construct the original $\psi(x, 0)$. Based on experience, $\epsilon$ is recognized to be of the same order as $\epsilon_{0}$. Finally, the candidate solution, $\hat{u}(x, t)$, is determined by evaluating Eq. (2) with the now complete set of parameters. The candidate solution is verified by numerical integration with the NLSE. Solutions are considered valid if the residual after numerical integration is small and decreases with grid refinement.

The solution can be analyzed in terms of its maximum amplitude defined as

$$
\gamma=A+2 \lambda_{I}
$$

where $A=1$ for all cases presented in this work. Solutions with maximum amplitudes even slightly greater than the background level can be critically important in systems where extreme waves are of interest. The parameter map presented here allows for identification of candidate solutions with large maximum amplitudes through inspection of $\lambda_{I}$ values.

\section{Parameter map: Features and overview}

The map of graded parameters obtained for $L=$ 15 is shown in Figure 2. The map exhibits approximate horizontal and vertical symmetry, and discrete bands. The corrected eigenvalues, which are used to determine actual solutions, typically lie on or in the regions highlighted in the map. Predicted values from the band associated with $\lambda_{I}=0$ lead to stable Stokes wave solutions. Solutions in other bands exhibit growth. The solution bands have an upper bound that depends on $L$. Other solutions can exist away from the bands, as isolated points.

A singularity exists in the mapped space at $(0, i)$. This eigenvalue is associated with the Peregrine solution, a homoclinic solution in which the temporal and spatial periods both $\rightarrow \infty$. The eigenvalue procedure detailed above was developed for near homoclinic solutions and this procedure is not capable of

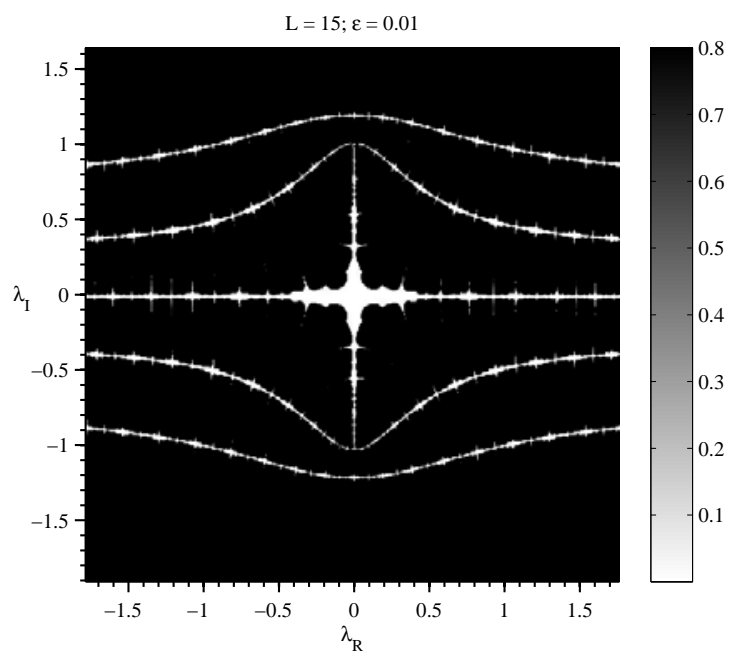

Figure 2: Parameter map for $L=15$.

resolving the single eigenvalue associated with a homoclinic solution such as the Peregrine. However, near homoclinic solutions exist near $(0, i)$ and are referred to as near Peregrine solutions. Such solutions were addressed for the first time in reference [25]. These solutions appear to be quite similar to the Peregrine solution, but demonstrate quasiperiodic fluctuations. The solutions also tend to decrease in residual error as $L$ increases, which is consistent with the spatial and temporal support of the Peregrine solution.

One example of a near Peregrine solution corresponding to $\lambda_{R}=0.0098, \lambda_{I}=1.0068, \epsilon=$ $0.0075, \theta=-0.3617$, and $L=15$ with residual error $\rho=0.04 \%$ is examined more closely in Figure 3 . The $\lambda_{i}$ values are extremely close to $(0, i)$, but remain distant enough to be resolved through the eigenvalue solution procedure. The solution exhibits similar features to the peak of the Peregrine solution such as the peak amplitude and decay profile. This solution is compared to the exact Peregrine solution in Section 4.2.

\section{Rogue-wave solutions}

The parameter map reveals the presence of many solutions with eigenvalues far from those associated with the Peregrine form (far from $\lambda=(0, i))$. One solution with eigenvalues $\lambda=(1.1367,1.2076 i)$ is chosen as a representative example. The remaining spectral parameters of the solution are $\epsilon=0.00416$ and $\theta=0.37202$. This solution satisfies the original NLSE quite well with an estimated error of 


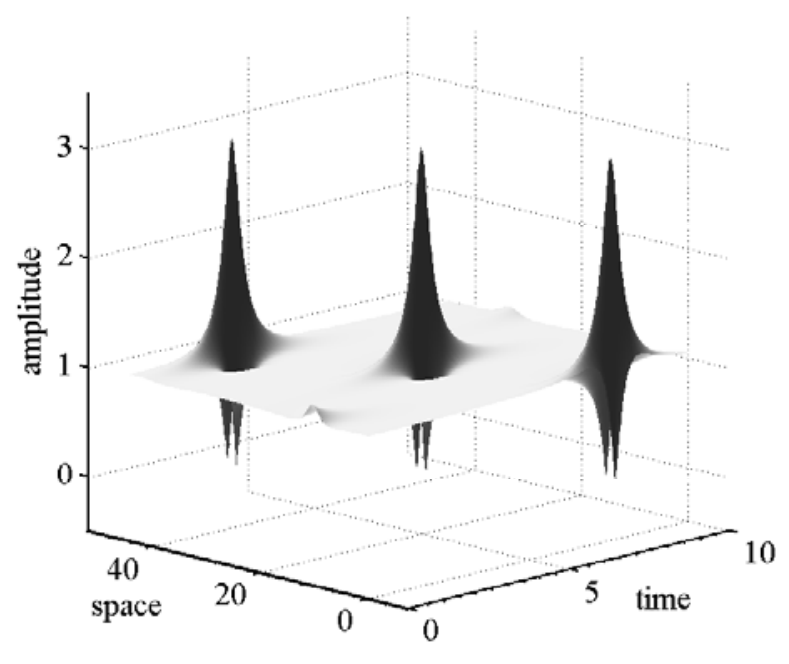

Figure 3: Near homoclinic solution with eigenvalues $\left(\lambda_{R}=\right.$ $\left.0.0098, \lambda_{I}=1.0068\right)$ close to $\left(\lambda_{R}, \lambda_{I}\right)$ associated with the Peregrine solution of the NLSE.

$\rho=0.009 \%$. Rogue-wave like behavior is exhibited as the peak is 3.4 times the background envelope consistent with Eq. (11), as seen in Figure 4. This solution is revisited in Section 4.2.

\subsection{Transition from rogue wave to exaggerated wave}

As a final feature of the parameter map profiled in this paper, the qualitative transition of a rogue wave to a wave with less pronounced undulation is discussed. The direct relationship between the wave amplitude and the value of $\lambda_{I}$ is provided by Eq. (11). The amplitude decreases along with the $\lambda_{I}$ value. As the spectral parameter $L$ increases, the solution bands tend to compress towards the real axis, resulting in decreased $\lambda_{I}$. For $L=[6,8, \ldots, 14]$, solutions from the highest band of each solution space near the imaginary axis $\left(\lambda_{R} \approx 0\right)$ are compared. A composite map containing only the top branch of the predicted space for the given values of $L$ is shown in Figure 5. The corrected $\left(\lambda_{R}, \lambda_{I}\right)$ pairs of the progression are indicated with a white colored ' $\mathrm{x}$ ' in the figure.

A description similar to Eq. (11) for the value of the wave's maximum saddle has been determined as $A_{\text {saddle }}=2 \lambda_{I}-1$. Stated differently, and combined with Eq. (11), the wave's maximum is always two units higher than the saddle point. This relationship was found to be consistent for solutions throughout the $\lambda$ plane, including the Pere-

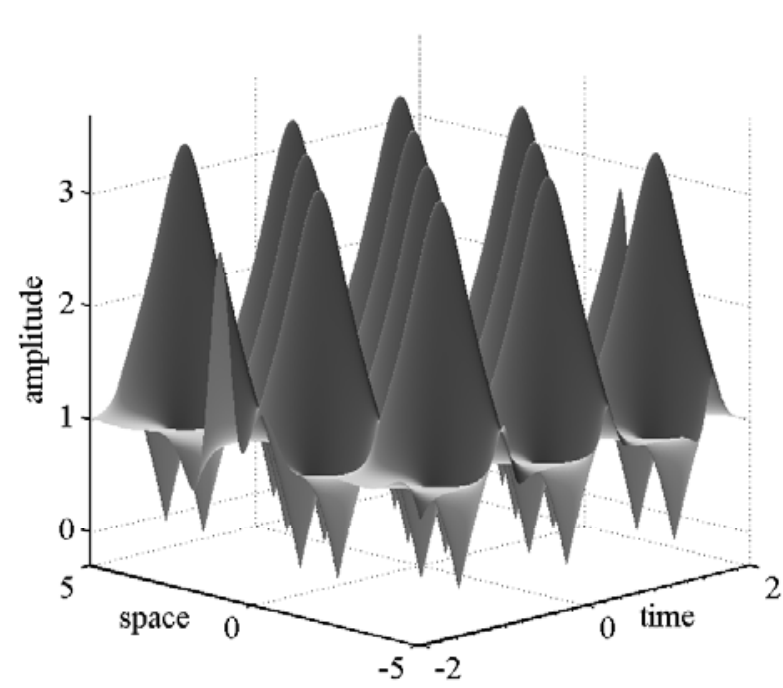

Figure 4: Representative scaled rogue-wave solution with $\lambda=(1.1367,1.2076 i)$

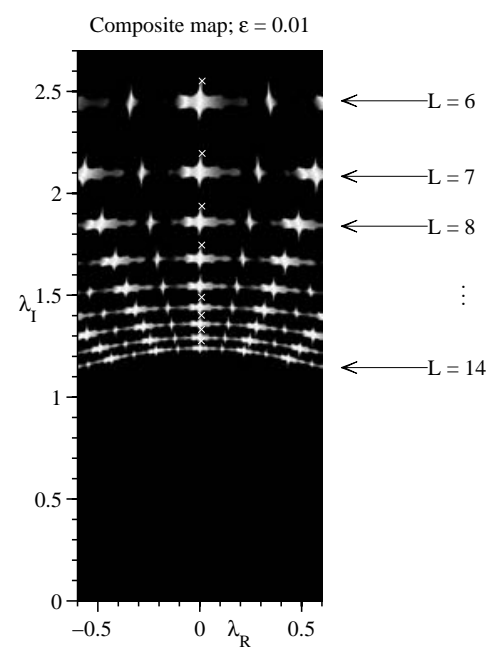

Figure 5: Composite parameter map containing the highest solution band for multiple values of L. Corrected pairs of $\left(\lambda_{R}, \lambda_{I}\right)$ for generated solutions are marked with an ' $\mathrm{x}$ '. 


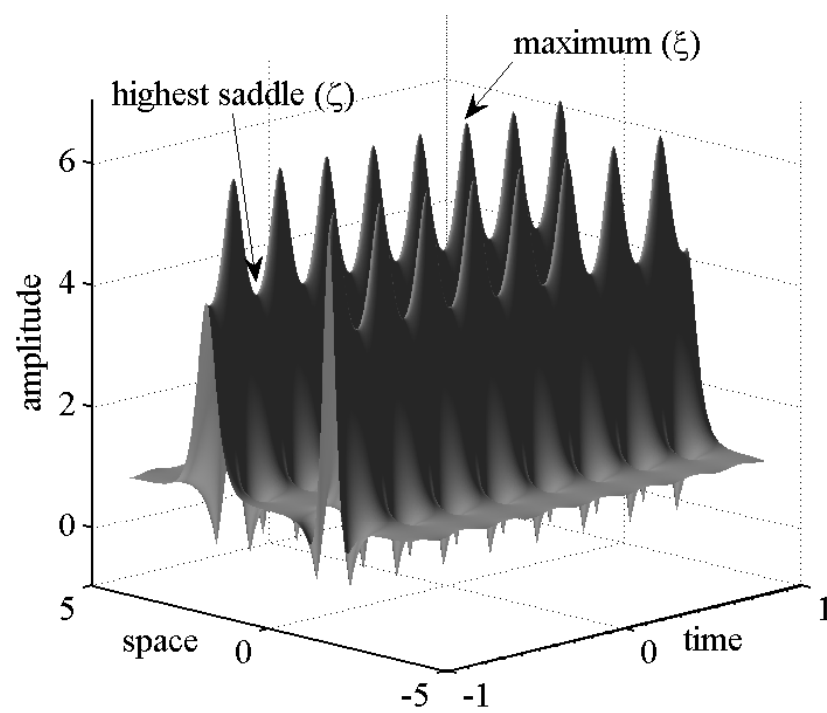

Figure 6: Peaked rogue wave: $L=6 ; \lambda=0.01178+2.5512 i$; and $A_{\max }=6.1$

grine breather solution and solutions with $\lambda_{I}<1$. The theoretical origin of this relationship and its required conditions will be considered in a future effort. This feature is illustrated with several solutions beginning with a solution on the highest branch of $L=6$ as depicted in Figure 6.

Solutions from the top branch for several different values of $L$ are shown in Figure 7 . In the progression, as $L$ is increased the values of $\lambda_{I}$ decrease. A solution chosen from the next higher value of $L$ (lower $\lambda_{I}$ ) in Figure 5 exhibits a lower maximum saddle, and thus a smaller difference from the saddle to the background. Progressing down the branches of the predictive map, the maximum saddle for a solution is found to decrease and the undulations of the peaks become more prominent as shown in the collection of figures. The Peregrine solution represents a limiting case where the maximum saddle and the background are coincident (i.e., 1) and $L \rightarrow \infty$.

\subsection{Physical scaling}

Solutions can be rescaled to physically meaningful dimensions following the notation and procedure presented in earlier work [1]. With the time and space coordinates scaled as
Table 1: Summary of the spectral parameters for the rogue wave solutions from the upper most band with $\lambda_{R} \approx 0$ for $L$ as indicated.

\begin{tabular}{c|c|c|c|c|} 
case & $L$ & $\left(\lambda_{R}, \lambda_{I}\right)$ & $\epsilon$ & $\theta$ \\
\hline 1 & 6 & $(0.0118,2.551)$ & 0.00259 & 0.0826 \\
2 & 8 & $(0.0107,1.937)$ & 0.00252 & 0.1159 \\
3 & 10 & $(0.0061,1.549)$ & 0.00259 & 0.1584 \\
4 & 12 & $(0.0086,1.402)$ & 0.00260 & 0.1874 \\
5 & 14 & $(0.008,1.276)$ & 0.00267 & 0.2359
\end{tabular}

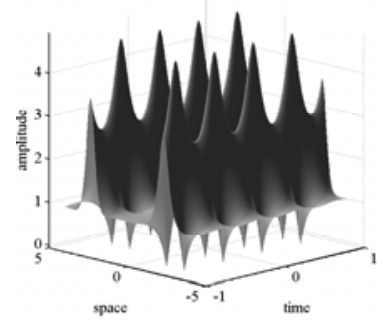

(a) Case 2: $A_{\max }=4.87$

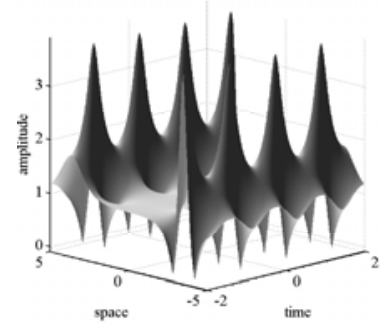

(c) Case 4: $A_{\max }=3.8$

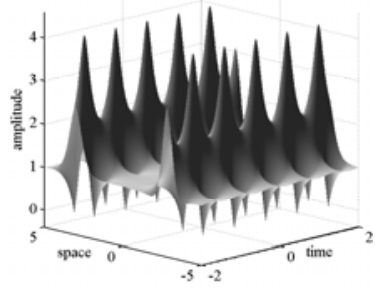

(b) Case 3: $A_{\max }=4.2$

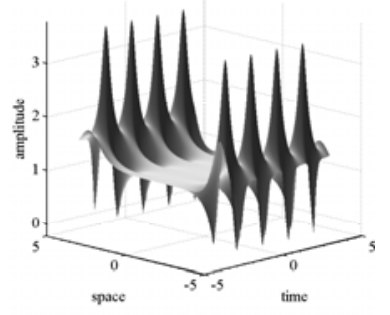

(d) Case 5: $A_{\max }=3.55$
Figure 7: Example rogue waves corresponding to cases shown in Table 1.

$T=-\frac{k_{0}^{2} a_{0}^{2} \omega_{0}}{4} t, \quad X=\sqrt{2} k_{0}^{2} a_{0}\left(x-\frac{\omega_{0}}{2 k_{0}} t\right), \quad \psi=a_{0} u$

the solutions satisfy

$$
i\left(\psi_{t}+\frac{\omega_{0}}{2 k_{0}} \psi_{x}\right)-\frac{\omega_{0}}{8 k_{0}^{2}} \psi_{x x}-\frac{\omega_{0} k_{0}^{2}}{2}|\psi|^{2} \psi=0
$$

where $t$ and $x$ are time and spatial coordinates, and $k_{0}$ is the wave number, $\omega_{0}$ is the carrier wave frequency, and $\eta(x, t)=\operatorname{Re}\left[\psi(x, t) \exp \left[i\left(k_{0} x-\omega_{0} t\right)\right]\right.$ is the surface elevation.

Theta function solutions are expressed as 


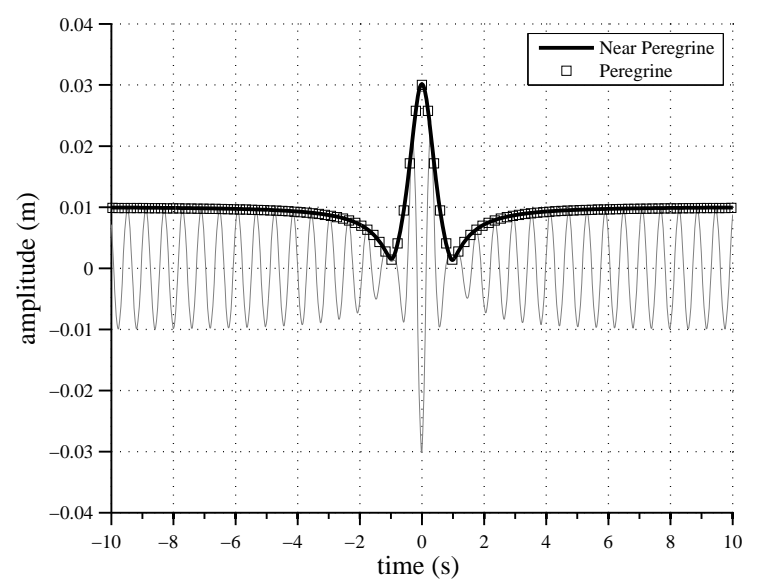

Figure 8: The near Peregrine solution (solid line) appears identical to the Peregrine solution (marked with squares) over the interval examined.

$$
\psi(x, t)=a_{0} \frac{\Theta\left(x, t \mid \tau, \delta^{-}\right)}{\Theta\left(x, t \mid \tau, \delta^{+}\right)} e^{-\frac{k_{0}^{2} a_{0}^{2} \omega_{0}}{2} t} .
$$

where $\Theta\left(x, t \mid \tau, \delta^{ \pm}\right)$is determined by substituting Eq. (12) in Eq. (3).

The rescaling procedure with $\left(a_{0}=0.01 \mathrm{~m}, \omega_{0}=\right.$ $10.7 \mathrm{~s}^{-1}, k_{0}=11.63 \mathrm{~m}^{-1}$ ) [1] was applied to the near Peregrine case of Figure 3, phase shifted to align its peak location to $t=0$. It is compared to the exact Peregrine case obtained in prior work [1]. The two solutions are almost identical, as shown along with the surface elevation in Figure 8. Both solutions exhibit similar maxima and minima. Over a larger domain, the periodic nature of the near Peregrine solution would become evident.

\subsection{Evolution of a dimensional wave field}

The evolution of a wave field governed by the NLSE can be evaluated based on the previously generated solutions. The temporal fluctuations observed at several fixed spatial locations in the wave field of the dimensionalized near Peregrine case defined in Eq. (14) are illustrated in Figure 9. The wave field serves as a validation for the solution and rescaling procedure as it was designed to be similar to the wave field presented in prior work [1]. In the present case, the near Peregrine rogue wave achieves a maximum at 128 seconds $8.2 \mathrm{~m}$ downstream from the datum.

Additional insights can be gained by studying the wave field of a solution with eigenvalues quite different from those of the Peregrine. In this case,

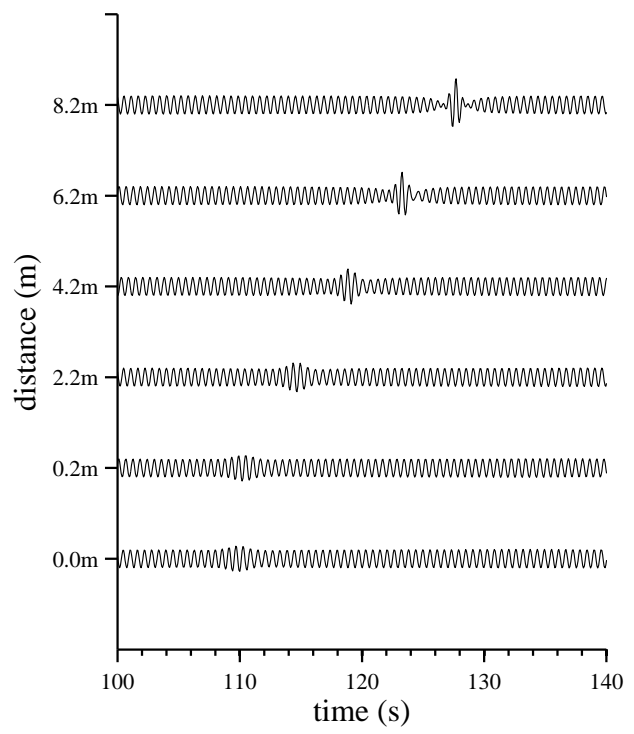

Figure 9: Predicted temporal evolution for a periodic noneven solution $\left(\lambda_{R}=0.0098, \lambda_{I}=1.0068\right)$. Surface heights are shown at various distances.

physical parameters that match the water tank used previously in [11], namely, $a_{0}=0.04 m, \omega_{0}=$ $4.8308 \mathrm{~s}^{-1}$, and $k_{0}=2.3813 \mathrm{~m}^{-1}$, were applied to rescale the solution presented in Figure 4, for a similar wave field analysis.

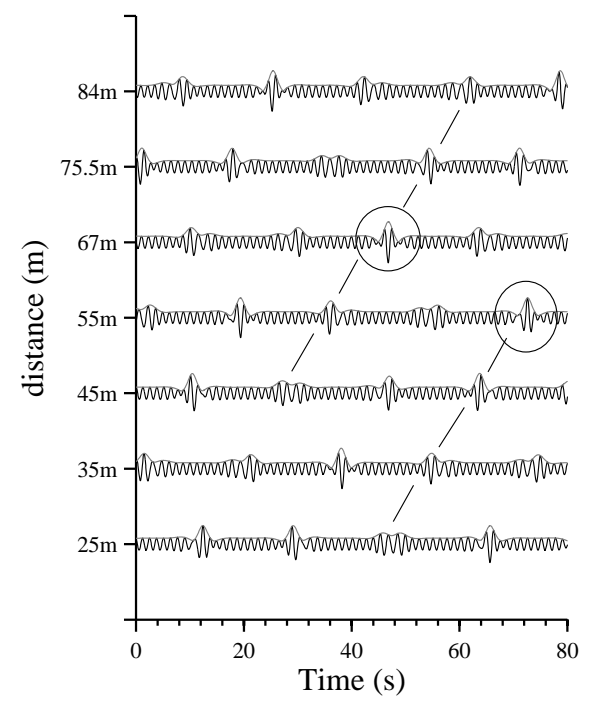

Figure 10: A wave field showing the predicted temporal evolution of a rogue-wave solution $\left(\lambda_{R}=1.1367, \lambda_{I}=1.2076\right)$ with two localization events highlighted.

The wave field evolution contains several wave packets that mutually interfere, as shown in Fig- 


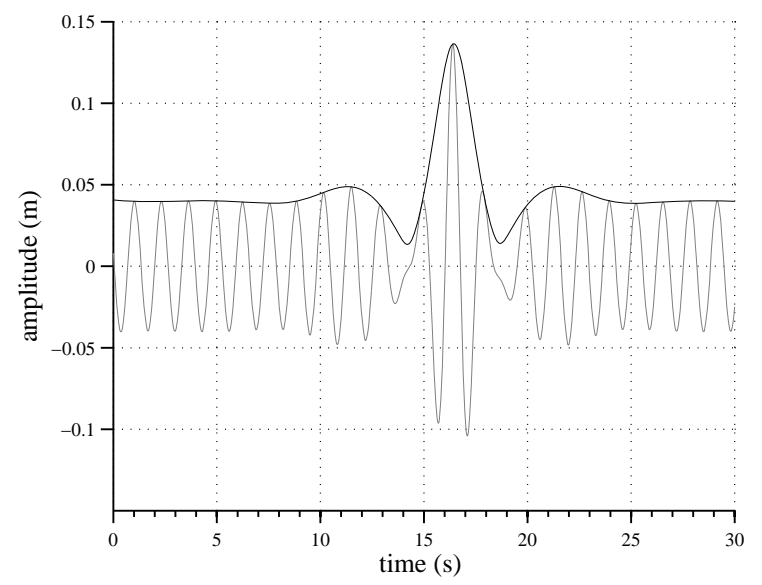

Figure 11: Detailed view of dimensional rogue-wave solution with $\lambda=(1.1367,1.2076 i)$.

ure 10. The spatio-temporal localization of wave energy of one of the wave packets is highlighted in the figure at $(t=45 \mathrm{~s}$, distance $=67 \mathrm{~m})$. The evolution of this localization can be tracked from a perturbation with two distinct peaks close to $(t=28 s$, distance $=45 m)$. This fluctuation localizes into a single large fluctuation at the time highlighted, and subsequently delocalizes at a later time. An additional evolution is highlighted beginning at $(t=48 s$, distance $=25 \mathrm{~m})$. A similar localization takes place as the wave packets convect in time and space. The localization of maximal amplitude can be identified at approximately $(t=72 s$, distance $=55 \mathrm{~m})$.

A detailed view of the surface height and envelope of the first localization is shown in Figure 11. While this solution appears to have a similar character to a Peregrine breather, its eigenvalues are quite different, and its peak is more than 3 times the surface height, higher than that of the Peregrine solution. This solution is one of many that could be used to gain insights into the degree to which waves in a medium can be modeled by the NLSE. Since this wave field is a solution to the NLSE, a system which is governed by the NLSE should be capable of propagating this solution as predicted. Furthermore, similar wave field evolutions could be used to predict a localization event based on a limited set of measurements at a single spatial location.

\section{Time-Frequency analysis}

The continuous wavelet transform, defined in [26] as

$$
X_{w}(a, \tau)=\frac{1}{\sqrt{a}} \int_{-\infty}^{\infty} x(t) \bar{\psi}\left(\frac{t-\tau}{a}\right) d t
$$

where $X_{w}$ are the complex wavelet coefficients, $a$ is the wavelet scale, $\tau$ is the wavelet time, $x(t)$ is the signal under consideration, and $\Psi(t)$ is the mother wavelet. This transform has been applied in many fields to analyze the time-frequency content in unsteady signals (e.g., [27]). Choosing the Morlet wavelet, defined as

$$
\psi(t)=\frac{1}{\sqrt{\pi F_{b}}} e^{2 \pi i F_{c} t} e^{-\frac{t^{2}}{F_{b}}}
$$

in [28] with $F_{b}=2$ and $F_{c}=1.75$ provides a balance between frequency resolution and time resolution. Based on the above definition, the wavelet scale, $a$, can be thought of in terms of the wavelet frequency $f_{\text {wavelet }}=a / F_{c}$. With this choice of wavelet basis, the time evolution of the Peregrine solution can be examined in the time-frequency domain. In the wavelet energy spectrum defined as [26]

$$
W E S(a, \tau)=\frac{X_{w}(a, \tau) X_{w}^{*}(a, \tau)}{a}
$$

the frequency bands of the wavelet coefficients are normalized so that the amplitude of $W E S(a, \tau)$ is equal for pure sine waves at all frequencies under consideration.

Time-frequency analyses of the temporal histories of the Peregrine solution at three fixed locations in space are carried out. The spatial locations have been chosen to correspond to the wave before, during, and after its maximum amplitude. This record can be considered as a simulation of experimental histories, that are typically recorded at fixed spatial locations. The first case analyzed is the Peregrine solution before it has reached its maximum amplitude. The presence of energy can be identified in the carrier wave of the Peregrine solution as seen near $1.75 \mathrm{~Hz}$ in Figure 12. Energy transfer into bands slightly above and below the carrier can be identified as indicated by the large responses in $W E S(a, \tau)$ near $1.5 \mathrm{~Hz}$ and $2 \mathrm{~Hz}$ at 77 seconds and the diminished response in the carrier wave around that time.

The time of maximum amplitude also corresponds to maximum energy transfer into the side 


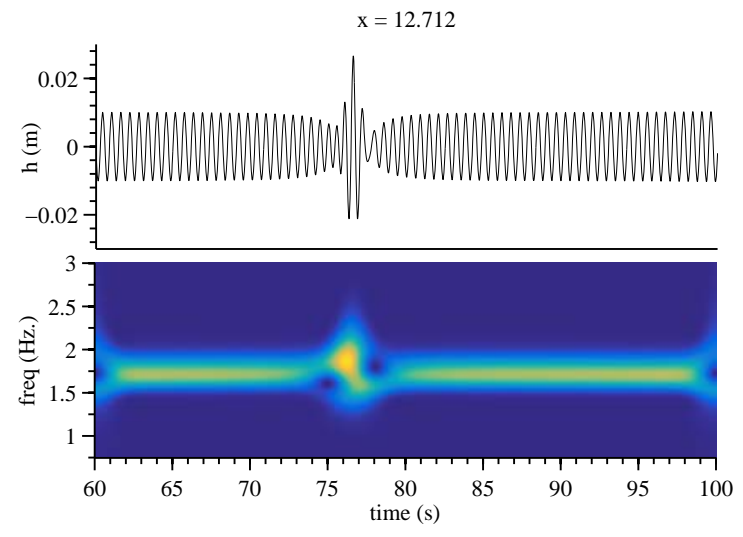

Figure 12: $W E S(a, \tau)$ of the Peregrine solution before its peak amplitude is reached.
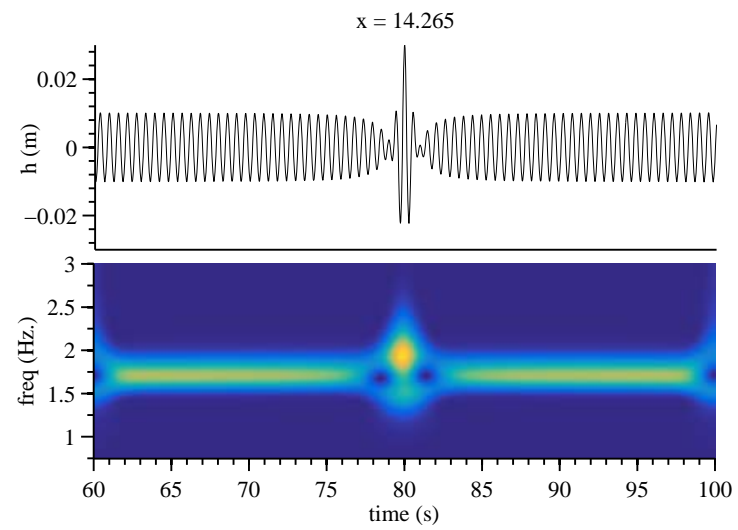

Figure 13: $W E S(a, \tau)$ of the Peregrine solution at its peak amplitude.

bands centered around $1.5 \mathrm{~Hz}$ and $2.0 \mathrm{~Hz}$ at $t=80$ seconds, as seen in Figure 13. In this case, more energy is transferred into the higher frequency band than the lower frequency band. Finally, energy is in the process of being transferred from the side bands back into the carrier wave as seen in the final frame of the evolution shown in Figure 14. A complete animation of the sequence is provided in an electronic appendix.

The dispersion relation can be identified through the sequence of presented snapshots. As seen in Figure 12, energy in the lower frequencies initially lags that of the higher frequency band; that is, the lower frequency band appears at a fixed spatial location at a later time than energy in the higher frequency bands. During and after the interference (i.e., Figures 13 and 14), energy in the lower frequency band appears at the given spatial location

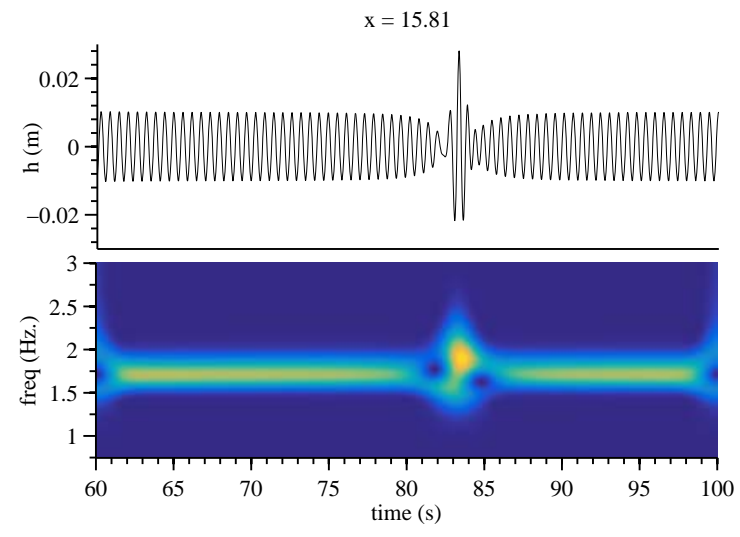

Figure 14: $W E S(a, \tau)$ of the Peregrine solution just after its peak amplitude has been reached.

at the same time and at an earlier time than that of the higher frequency band. Energy in the lower frequency band requires less time to travel the same distance as energy in the higher band; consistent with the dispersion relation for deep water.

The solution presented in Figure 10 is examined with a similar analysis. In this case, a complete evolution can be identified in a single snapshot; however, several snapshots are provided. Again, significant energy can be identified in the carrier band of $0.75 \mathrm{~Hz}$, as shown in Figure 15. At the first spatial location analyzed, a wave with maximum amplitude can be identified at $t=45$ seconds. Its energy is separated into lower and upper side bands around $0.6 \mathrm{~Hz}$ and $0.9 \mathrm{~Hz}$, respectively. At a further spatial location, the peak has moved to $t=55$ seconds somewhat altered from its initial state. Energy can be identified merging with the carrier frequency, and lower frequency components arrive at the spatial location ahead of higher frequencies. Finally, in the third spatial location, the energy has transferred almost completely from the upper side band at $t=60$ seconds, as shown in Figure 17. Again the dispersion relation is evident as the frequencies in the lower side band in each wave packet travel faster. A complete animation of this sequence is provided in an online appendix.

\subsection{Reconstruction}

The inverse continuous wavelet transform (ICWT) can be defined as [26] 


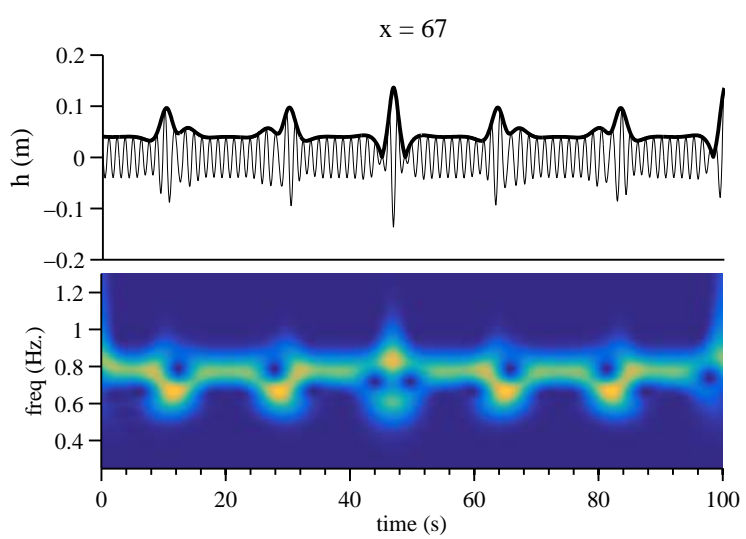

Figure 15: $W E S(a, \tau)$ of the wave field shown in Figure 10, illustrative of a maximum amplitude wave.

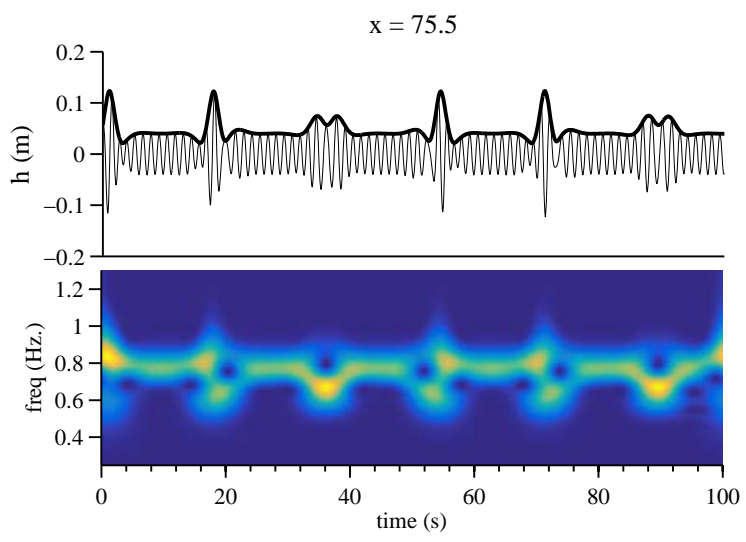

Figure 16: $W E S(a, \tau)$ of the wave field shown in Figure 10 with the large amplitude wave diminished.

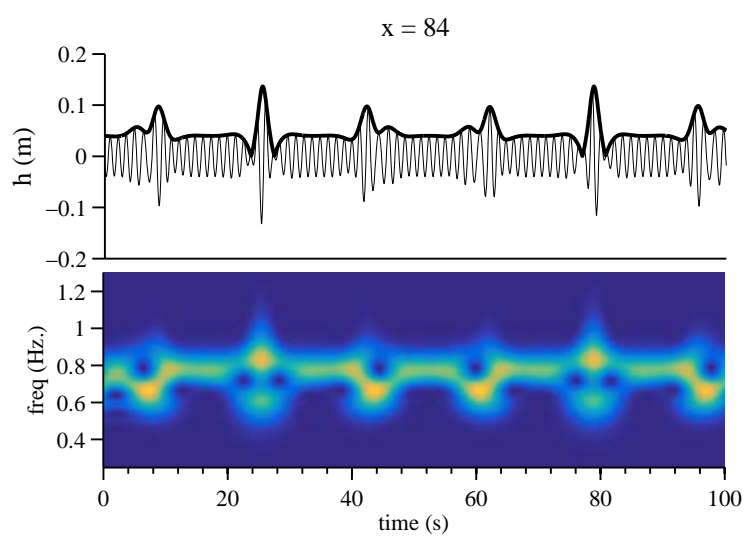

Figure 17: $W E S(a, \tau)$ of the wave field shown in Figure 10 with the large amplitude wave significantly diminished.

$$
\begin{aligned}
& x(t)= \\
& \frac{1}{C_{\Psi}} \int_{0}^{\infty} \frac{1}{a^{2}} \int_{-\infty}^{\infty} X_{w}(a, \tau) \frac{1}{\sqrt{a}} \Psi\left(\frac{t-\tau}{a}\right) d \tau d a
\end{aligned}
$$

with

$$
C_{\Psi}=\int_{0}^{\infty} \frac{|\hat{\Psi}(\omega)|^{2}}{\omega} d \omega
$$

In this construction, the original signal $x(t)$ is reconstructed based on the wavelet coefficients and the analyzing wavelet. The ICWT can be used to reconstruct the signal over a selected frequency band by integrating over only those scales, $a$, which are associated with the frequencies of interest. This type of selective reconstruction can be used to provide insights into time varying energy transfer in a given band. For this analysis, wavelet parameters of $F c=1.7506, F b=5$ were chosen for finer frequency resolution with slightly relaxed temporal resolution. Treating the Peregrine solution at its maximum amplitude $(x=14.265)$, yields the time varying energy contained in three bands chosen as band $_{1}=[1.00,1.58) \mathrm{Hz}$ which spans the lower side band, band $_{2}=[1.58,1.86] \mathrm{Hz}$, which spans the carrier wave, and band $_{3}=(1.86,3] \mathrm{Hz}$ which spans the upper side band, as shown in Figure 18. The energy in the upper and lower side bands clearly experiences a sharp rise along with the increase in the Peregrine amplitude. The energy in the carrier frequency diminishes; however, some residual response remains at the peak time of 80 seconds. The analysis was validated by combining the three signals, which yielded the original Peregrine wave to within working precision.

A similar reconstruction was performed for the more complex wave field shown in Figure 10. In this case, bands were chosen as band $1=[0.50,0.72)$ $\mathrm{Hz}$, band $_{2}=[0.72,0.79] \mathrm{Hz}$, band $_{3}=[0.79,1.50)$ $\mathrm{Hz}$ to isolate the energy transfer as much as possible. The maximum surface amplitude occurs at around 47 seconds in the wave field record. At the maximum surface amplitude, the upper most band shows a defined maximum, the carrier band shows an envelope minimum, and the lowest band shows a local envelope maximum. Overall, the envelope of the upper side band has a periodic character with repetitions every 20 seconds. However, at the surface height peak event, the elapsed time between the band envelope maximum and adjacent envelope maximum is slightly decreased. The envelope 

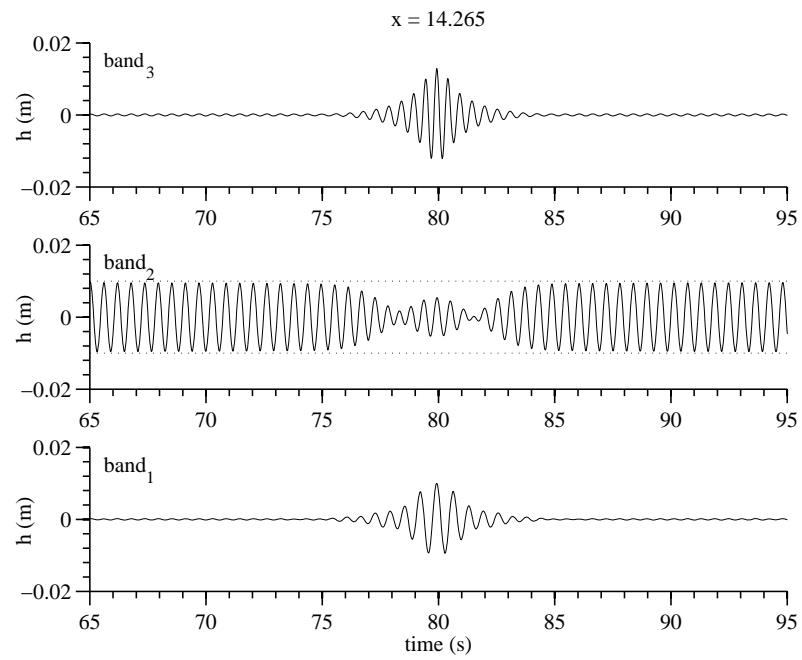

Figure 18: ICWT of the Peregrine solution at $(x=14.265)$ showing maximum energy transfer into the upper and lower side bands.

of the carrier band maintains a periodic character with about 15 seconds between envelope maxima. The time is elongated to approximately 25 seconds between the maxima that span the maximum surface height event. Finally, the envelope of the lower side band maintains a periodic character at an interval just under 20 seconds. At the maximum surface height event, the amplitude of the band envelope experiences a local maximum, that is actually slightly lower than the periodic maxima through the remaining record.

\section{Concluding remarks}

An eigenvalue prediction and solution scheme, which can be used to determine single mode near homoclinic theta function based solutions to the nonlinear Schrödinger equation has been described. This procedure facilitates the determination of regimes of known extreme wave solutions such as the Peregrine breather, as well as periodic rogue waves that exhibit localizations similar to the Peregrine and waves that behave qualitatively differently. The studies also reveal an interesting geometric relationship between the saddle point and the maximum height for the rogue waves generated by this procedure. These wave fields can serve as the basis for experimental investigations into the degree to which extreme waves in a medium can be modeled by the NLSE. Continuous wavelet analysis can
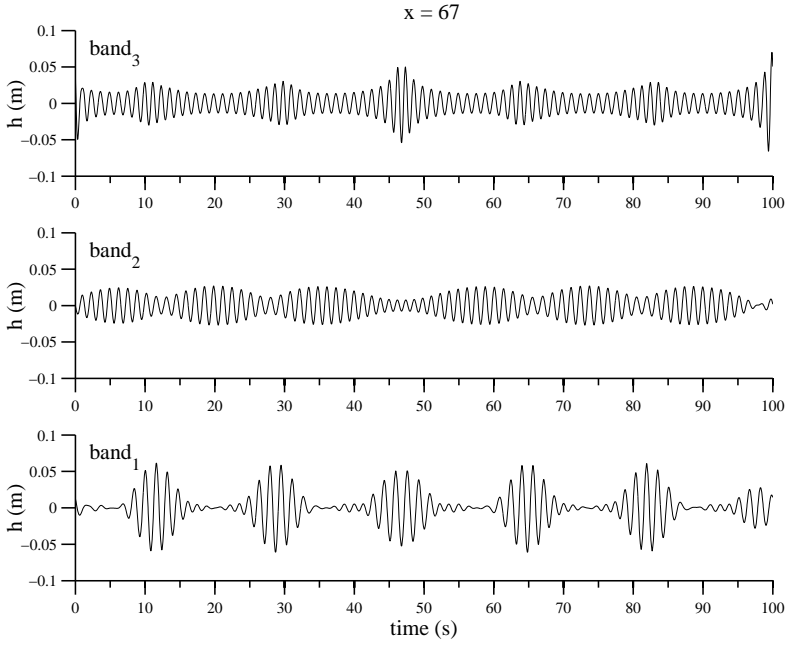

Figure 19: ICWT of the solution shown in Figure $10(x=67)$ showing maximum energy transfer for a peak event.

be used to observe and characterize intermittent energy exchange among the carrier, upper, and lower side bands. This type of analysis can be useful for many systems that experience Benjamin-Fier type instabilities. The inverse continuous wavelet transform can selectively reconstruct the carrier and side bands to further identify intermittent energy transfer amongst them. The Morlet wavelet is noted to be an appropriate choice of wavelet basis for the observation and reconstruction of side band energy transfer.

\section{Acknowledgments}

Support received for this work through NSF Grant No. CMMI-1125285 is gratefully acknowledged.

\section{References}

[1] A. Chabchoub, N. P. Hoffmann, N. Akhmediev, Phys. Rev. Lett. 106 (2011) 204502. URL: http://link.aps.org/doi/10.1103/PhysRevLett.106.204502. doi:10.1103/PhysRevLett.106.204502.

[2] A. Chabchoub, N. Hoffmann, M. Onorato, N. Akhmediev, Phys. Rev. X 2 (2012) 011015. URL: http://link.aps.org/doi/10.1103/PhysRevX.2.011015. doi:10.1103/PhysRevX.2.011015.

[3] D. Solli, C. Ropers, P. Koonath, B. Jalali, Nature 450 (2007) 1054-1057.

[4] A. R. Osborne, M. Onorato, M. Serio, Physics Letters A 275 (2000) 386-393.

[5] T. B. Benjamin, J. Feir, J. Fluid Mech 27 (1967) 417430. 
[6] C. Kharif, E. Pelinovsky, European Journal of Mechanics-B/Fluids 22 (2003) 603-634.

[7] A. Chabchoub, N. Hoffmann, N. Akhmediev, Journal of Geophysical Research: Oceans (1978-2012) 117 (2012).

[8] M. Onorato, A. Osborne, M. Serio, L. Cavaleri, Physics of Fluids (1994-present) 17 (2005) 078101.

[9] M. Onorato, A. Osborne, M. Serio, L. Cavaleri, C. Brandini, C. Stansberg, European Journal of Mechanics-B/Fluids 25 (2006) 586-601.

[10] M. Onorato, A. Osborne, M. Serio, L. Cavaleri, C. Brandini, C. Stansberg, Physical Review E 70 (2004) 067302 .

[11] A. Osborne, Nonlinear Ocean Waves \& the Inverse Scattering Transform, volume 97, Access Online via Elsevier, 2010.

[12] K. Dysthe, H. E. Krogstad, P. Müller, Annu. Rev. Fluid Mech. 40 (2008) 287-310.

[13] C. Chabalko, A. Moitra, B. Balachandran, Physics Letters A 378 (2014) 2377-2381.

[14] E. R. Tracy, H. H. Chen, Phys. Rev. A 37 (1988) 815-839. URL: http://link.aps.org/doi/10.1103/PhysRevA.37.815. doi:10.1103/PhysRevA.37.815.

[15] N. Akhmediev, V. Korneev, Theoretical and Mathematical Physics 69 (1986) 1089-1093.

[16] A. Calini, C. M. Schober, Physics Letters A 298 (2002) 335-349.

[17] A. Islas, C. M. Schober, Physics of Fluids 17 (2005) 1-4.

[18] A. Osborne, The European Physical Journal-Special Topics 185 (2010) 225-245.

[19] B. Kibler, J. Fatome, C. Finot, G. Millot, F. Dias, G. Genty, N. Akhmediev, J. M. Dudley, Nature Physics 6 (2010) 790-795.

[20] H. C. Yuen, B. M. Lake, Annual Review of Fluid Mechanics 12 (1980) 303-334.

[21] V. Ruban, Y. Kodama, M. Ruderman, J. Dudley, R. Grimshaw, P. McClintock, M. Onorato, C. Kharif, E. Pelinovsky, T. Soomere, et al., The European Physical Journal-Special Topics 185 (2010) 5-15.

[22] O. Its, V. Kotliarov, Akademiia Nauk Ukrains koi RSR Dopovidi Seriia Fiziko Matematichni ta Tekhnichni Nauki 1 (1976) 965-968.

[23] I. M. Krichever, Russian Mathematical Surveys 32 (1977) 185-213.

[24] A. H. Nayfeh, B. Balachandran, Applied nonlinear dynamics: Analytical, computational, and experimental methods, Wiley, 1995.

[25] M. Ablowitz, B. M. Herbst, C. M. Schober, Physica A (1996) 212-235.

[26] S. Mallat, A wavelet tour of signal processing, Academic press, 1999.

[27] C. C. Chabalko, Z. Ge, M. R. Hajj, W. A. Silva, Journal of aircraft 43 (2006) 1082-1088.

[28] A. Teolis, Computational signal processing with wavelets, Springer Science \& Business Media, 1998. 